\title{
Chain formation of metal atoms
}

Bahn, Sune Rastad; Jacobsen, Karsten Wedel

Published in:

Physical Review Letters

Link to article, DOI:

10.1103/PhysRevLett.87.266101

Publication date:

2001

Document Version

Publisher's PDF, also known as Version of record

Link back to DTU Orbit

Citation (APA):

Bahn, S. R., \& Jacobsen, K. W. (2001). Chain formation of metal atoms. Physical Review Letters, 87(26), 266101. https://doi.org/10.1103/PhysRevLett.87.266101

\section{General rights}

Copyright and moral rights for the publications made accessible in the public portal are retained by the authors and/or other copyright owners and it is a condition of accessing publications that users recognise and abide by the legal requirements associated with these rights.

- Users may download and print one copy of any publication from the public portal for the purpose of private study or research.

- You may not further distribute the material or use it for any profit-making activity or commercial gain

- You may freely distribute the URL identifying the publication in the public portal

If you believe that this document breaches copyright please contact us providing details, and we will remove access to the work immediately and investigate your claim. 


\title{
Chain Formation of Metal Atoms
}

\author{
Sune R. Bahn and Karsten W. Jacobsen \\ CAMP, Department of Physics, Technical University of Denmark, DK-2800 Kongens Lyngby, Denmark
} (Received 28 June 2001; published 4 December 2001)

\begin{abstract}
The possibility of formation of single-atomic chains by manipulation of nanocontacts is studied for a selection of metals ( $\mathrm{Ni}, \mathrm{Pd}, \mathrm{Pt}, \mathrm{Cu}, \mathrm{Ag}, \mathrm{Au})$. Molecular dynamics simulations show that the tendency for chain formation is strongest for Au and Pt. Density functional theory calculations indicate that the metals which form chains exhibit pronounced many-atom interactions with strong bonding in low coordinated systems.
\end{abstract}

DOI: 10.1103/PhysRevLett.87.266101

PACS numbers: 68.65.La, 61.46.+w, 62.25. $+\mathrm{g}$

The ultimately smallest electrical wire that one can imagine would consist of just a single metallic chain of atoms. Such atomic chains have recently been produced as freely suspended wires by manipulation of nanocontacts $[1,2]$. The formation of single-atomic chains consisting of metal atoms was first seen in molecular dynamics simulations $[3,4]$ and their existence has in the case of gold been experimentally confirmed with several techniques including mechanically controllable break junctions [1,5], high resolution transmission electron microscopy $[2,6]$, and combined scanning tunneling/atomic force microscopies [7]. Because of the quantum confinement of the electrons in the chains they exhibit interesting behavior with respect to their mechanical, electrical, and chemical properties as discussed in a number of studies both experimentally $[1,2,5,6]$ and theoretically $[4,8-12]$.

In the present Letter we address the question of the chain formation itself for a range of metals. From the outset it may seem rather surprising that it is possible to form atomic chains of a noble metal such as gold by mechanical means. After all, noble metals are known to energetically prefer close-packed structures where all the atoms are highly coordinated and the amount of directional bonding between gold atoms is very limited. In the following, we analyze the chain formation in more detail with two different methods. Using molecular dynamics simulations we study the breaking of contacts/wires of the late transition metals and noble metals $\mathrm{Ni}, \mathrm{Pd}, \mathrm{Pt}, \mathrm{Cu}, \mathrm{Ag}$, and $\mathrm{Au}$ and show that the tendency to chain formation is strongest for $\mathrm{Au}$ and Pt. Using density functional theory (DFT) we investigate the bond strength and breaking forces in chains and bulk structures. These studies also show that $\mathrm{Au}$ and $\mathrm{Pt}$ are characterized by strong bonds in chain structures. Frequent chain formation in $\mathrm{Au}$ and $\mathrm{Pt}$ seems to be in accordance with recent experimental investigations [5].

In the case of $\mathrm{Au}$, atomic chains can be readily formed in molecular dynamics simulations [4]. An example is shown in the upper row of Fig. 1 where the pulling of a small contact leads to the formation of a chain. The system is set up in a configuration formed by cutting out atoms from a regular fcc crystal with a total of 498 atoms in the contact. The axis of the contact is in the [110] direction. The atoms are moved using Langevin dynamics at a temperature of $4.2 \mathrm{~K}$ corresponding to typical (cryogenic) experimental conditions [1], and the contact is stretched at a rate of $1.92 \mathrm{~m} / \mathrm{s}$. For calculational reasons the rate is much faster than the experimental one, but it is slow enough for local atomic structures to relax. The interaction between the atoms is described with an effective-medium-theory (EMT) potential [13]. As seen in Fig. 1 the contact gradually gets thinner through a series of mechanical relaxations until a single-atom contact is obtained. At this stage atoms begin to be pulled out of the necks of the contact so that an atomic chain is formed. The pulling continues until the chain breaks, at which point we move the two parts together to form a new contact. After a few angstrom of indentation we start stretching again. The chain formation depends rather sensitively on the initial atomic configuration, as can be seen from the fact that although each successive indentation leads to qualitatively similar configurations in the beginning, some structures produce chains while others do not. In a series of 35 simulations with small variations in the initial configuration a chain was formed in 5 cases.

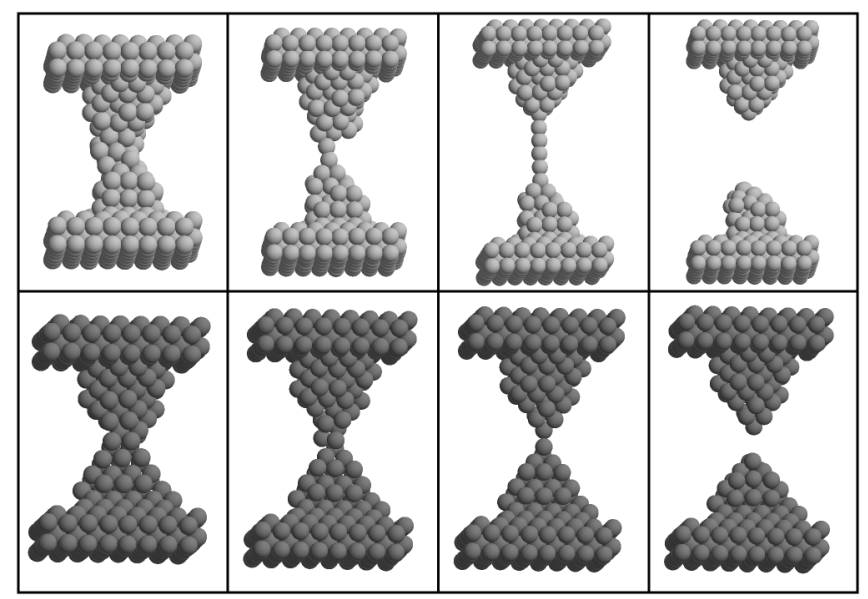

FIG. 1. Snapshots from simulations of breaking gold (upper row) and copper contacts (lower row). The gold contact exhibits the formation of a single-atomic chain, while the copper contact breaks after a single-atom contact is formed. Out of the six metals studied $(\mathrm{Cu}, \mathrm{Ag}, \mathrm{Au}, \mathrm{Ni}, \mathrm{Pd}$, and $\mathrm{Pt})$ only $\mathrm{Au}$ and $\mathrm{Pt}$ form chains. 
In order to investigate the tendency for different metals $(\mathrm{Ni}, \mathrm{Pd}, \mathrm{Pt}, \mathrm{Cu}, \mathrm{Ag}$, and $\mathrm{Au}$ ) to form atomic chains we perform similar simulations for the other five metals. The simulations start from an atomic configuration which is known to lead to the formation of a chain in the case of gold. The structure is rescaled according to the lattice parameter of the metal in question and relaxed. The contact is then stretched at the same conditions as for the gold contact. For all the investigated metals the initial stages are qualitatively similar and after a number of atomic restructurings a contact consisting of a single or a few atoms is formed. However, upon further stretching $\mathrm{Pt}$ is the only other metal for which an atomic chain is formed (4 times in a series of 36 stretchings). The $\mathrm{Ni}, \mathrm{Pd}, \mathrm{Cu}$, and $\mathrm{Ag}$ contacts break without chain formation as shown in the lower row of Fig. 1 for the case of $\mathrm{Cu}$. The strong tendency for $\mathrm{Au}$ and $\mathrm{Pt}$ to form chains is in agreement with recent experimental investigations [5]. The experiments also showed no chain formation for $\mathrm{Cu}$ and a weak tendency to chain formation in the case of $\mathrm{Ag}$ [5].

To understand the differences between the various metals we need to look closer at why chain formation is possible at all. Consider, for example, the simulated chain formation in gold, Fig. 1. The deformation involves a series of elastic stretching stages, each terminated by a discrete bond-breaking event [7]. Whether or not a chain is formed depends on which of the individual bonds break first, and therefore on the relative strength of bonds for different atomic configurations. The way a surface atom gets incorporated into a chain is by keeping the bond with a low coordinated chain atom while breaking the bonds to more highly coordinated atoms in the shoulders of the contact. The bond breakings occurring in the simulations usually happen one bond at a time, but sometimes more drastic changes can be observed, where the atom breaks two bonds to shoulder atoms more or less simultaneously.

Putting it simply, chain formation can occur if the bonds in the chain are much stronger than the bonds in the bulk so that it is harder to break the chain than to pull out an atom of the shoulders.

It is in fact typical for metallic bonding that the bond strength increases as the coordination number is reduced. This many-atom-interaction effect comes from the quantum mechanical bond formation and is, by construction, included in the EMT potential [13]. The bond strengthening for metals at low coordination numbers has also been demonstrated with DFT calculations $[14,15]$.

Out of the six metals $\mathrm{Ni}, \mathrm{Pd}, \mathrm{Pt}, \mathrm{Cu}, \mathrm{Ag}$, and $\mathrm{Au}$ the many-atom interactions play the largest role for $\mathrm{Au}$ and Pt [16]. Several interesting surface phenomena for $\mathrm{Au}$ and Pt can be explained on the basis of the strong variation of bond strength with coordination number. One example is the missing-row reconstruction of the (110) surfaces which occurs spontaneously for $\mathrm{Au}$ and $\mathrm{Pt}$, but not for the other four metals [16]. The many-atom interactions also play an important role for the tensile surface stress at metal surfaces and for reconstructions involving extended defects [17].

As mentioned the EMT potentials used in the simulations above include the important many-atom effects. On the other hand, the potentials are derived from mainly bulk properties [13] and it is therefore not clear that lowcoordinated systems such as atomic chains are appropriately described. Some properties of the chains may be understood on the basis of the "quantum well" states associated with the electronic motion perpendicular to the chains, and this quantized behavior is not accounted for in the potentials. It has, for example, been suggested that the calculated stability of gold chains in a zigzag structure [9] requires a detailed treatment of the electronic structure.

DFT is capable of describing electronic structure effects on the interatomic bonding, but due to limitations in computational speed it is not possible to directly study the chain formation by breaking of nanocontacts in the same way as with the interatomic potentials. However, it is possible to study the bond energies and forces in different simple structures and, as will be shown below, the DFT calculation confirm the trends in chain bond strength obtained with EMT.

In Fig. 2 we show the binding-energy curves for bulk fcc systems and for single-atomic chains calculated with DFT. The calculations are performed using periodic boundary conditions [18]. For the bulk calculations we use an fcc unit cell and the first Brillouin zone is sampled with 60 irreducible $\mathbf{k}$ points. The crystal is uniformly expanded and the calculated binding energies are divided by 6 to obtain the energy per nearest neighbor bond. For the chain calculations the unit cell also contains one atom so we consider only straight chains. The distance between neighboring chains is $11.7 \AA$ and the first Brillouin zone is sampled with eight $\mathbf{k}$ points along the chain. The size of the unit cell in the direction of the chain is varied to obtain the binding energy curve.

The results in Fig. 2 are obtained with non-spinpolarized calculations. Spinpolarized calculations show that for the straight chains considered here only $\mathrm{Ni}$ and Pt prefer a magnetic state and for Pt only when it is stretched quite far from the equilibrium distance. The effect of spinpolarization on the energetics is quite small and shall not be considered further here.

It is clear from Fig. 2 that the binding energy per bond is much larger in the chains than in the bulk systems giving a clear indication of many atom effects. For the bulk fcc systems the binding energy, $E_{0}$, at the equilibrium bond length is of the order $-1--0.4 \mathrm{eV}$ while for the chains the binding energies are as large as $-3--1 \mathrm{eV}$. The calculated binding energy curves can be fitted with the so-called "universal" binding energy curve [23] of the form

$$
E(x)=-\alpha\left(x-x_{0}\right) e^{-\beta\left(x-x_{0}\right)},
$$

where $x$ denotes the nearest neighbor bond length and where $\alpha, \beta$, and $x_{0}$ are fitting parameters. The three 


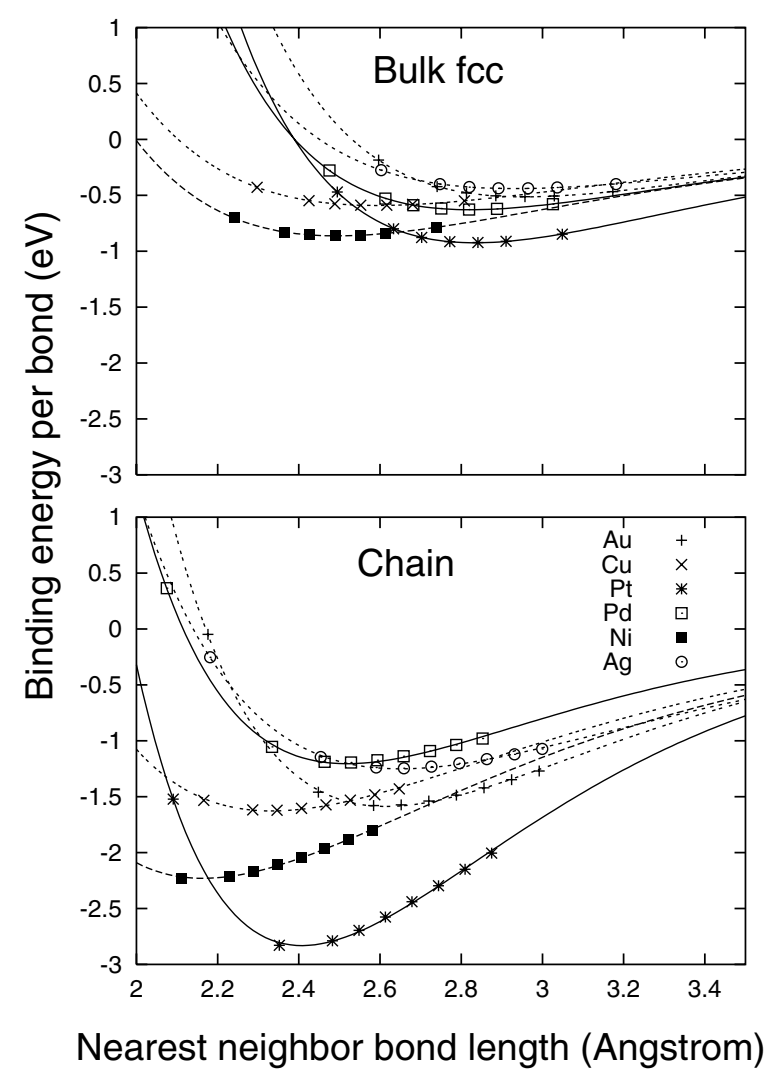

FIG. 2. Binding energy curves for bulk fcc crystals and straight atomic chains calculated within GGA. The bulk binding energy is divided by 6 to obtain the energy per nearest neighbor bond. The binding energies per bond in the chains are about 2-3 times that of the bulk fcc crystals. The fits have a rms deviation of $\lesssim 0.002 \mathrm{eV}$ for the bulk curves and $\lesssim 0.015 \mathrm{eV}$ for the chain.

parameters can be related to the equilibrium bond distance $d=x_{0}+1 / \beta$, the binding energy $E_{0}=-\alpha / \beta e$, and the slope at the inflection point $F_{0}=\alpha / e^{2}$. The resulting fits are quite good as can be seen from Fig. 2. The obtained fitting parameters are listed in Table I.

As discussed above the chain formation depends on how difficult it is to break bonds in the chain relative to other bonds in the regions close to the chain. It is possible to get an idea about how this varies from metal to metal from the calculated binding energy curves. As a measure of the force necessary to break a chain we can use the maximal force $F_{0}$ which we in the following shall call simply the break force. DFT calculations [7] of the breaking of gold chains of varying lengths and in different bonding geometries show that they break at forces which can be up to $25 \%$ lower than this ideal break force.

A rough estimate of the force necessary to break a "bulk"-like bond can be taken as the maximal slope of the binding energy curve for the fcc crystal. This bulk break force of course cannot be directly related to the yield stress of a real crystal which is dominated by complicated processes involving, for example, creation and mobility of dislocations. However, to study the trends for chain formation it will be sufficient.

Figure 3 shows the ratio of the break forces for the chains to the break forces of the bulk crystals for the six metals calculated with GGA and LDA. The break forces for the chains are seen to be generally 2-3 times larger than the bulk ones as is the case with the binding energy per bond. Au and Pt clearly stick out as the metals with the relatively largest break forces for the chains. Also for the binding energy per bond the chain/bulk ratio is particularly high for $\mathrm{Au}$ and Pt. This correlates nicely with the simulation results where chain formation is obtained only when breaking contacts of $\mathrm{Au}$ and $\mathrm{Pt}$.

The ratio of the break forces calculated with EMT is also shown in Fig. 3. The EMT results exhibit the same trends as the DFT results with high values for $\mathrm{Au}$ and $\mathrm{Pt}$, but the absolute values are about 50\% too high compared with the DFT values. The high values reflect that the break forces for the ideal chain geometry are too large when calculated with the EMT. However, this deficiency of the interatomic potential is to some extent counteracted by large relaxation effects. In an EMT simulation of the breaking of a chain, the chain breaks well before the inflection point of the binding energy curve and at a considerably smaller force. To illustrate this we have also included calculated break forces for fully relaxed systems which more closely reassemble the situation from the simulations of the full contact [24]. The trend with strong break forces in the chains for $\mathrm{Au}$ and $\mathrm{Pt}$ is unchanged by the relaxation and the value of the ratio is in much better agreement with the (unrelaxed) DFT results. Calculations for Au [7] show that the relaxation effects on the chain break force is much smaller with DFT than with EMT, and we therefore also expect a considerably smaller relaxation effect on the break force ratio with DFT.

TABLE I. Calculated parameters for the binding energy curves in Fig. 2: equilibrium bond distance $d$, equilibrium binding energy $E_{0}$ per bond, and break force $F_{0}$.

\begin{tabular}{lcrrrrrr}
\hline \hline Element & & \multicolumn{1}{c}{$\mathrm{Cu}$} & \multicolumn{1}{c}{$\mathrm{Ag}$} & \multicolumn{1}{c}{$\mathrm{Au}$} & $\mathrm{Ni}$ & \multicolumn{1}{c}{$\mathrm{Pd}$} & $\mathrm{Pt}$ \\
\hline \multirow{2}{*}{ Bulk fcc } & $d(\AA)$ & 2.59 & 2.93 & 2.96 & 2.49 & 2.82 & 2.83 \\
& $E_{0}(\mathrm{eV})$ & -0.59 & -0.44 & -0.51 & -0.86 & -0.63 & -0.93 \\
& $F_{0}(\mathrm{eV} / \AA)$ & 0.44 & 0.34 & 0.44 & 0.64 & 0.54 & 0.77 \\
Chain & $d(\AA)$ & 2.33 & 2.65 & 2.62 & 2.16 & 2.52 & 2.41 \\
& $E_{0}(\mathrm{eV})$ & -1.63 & -1.25 & -1.59 & -2.23 & -1.20 & -2.83 \\
& $F_{0}(\mathrm{eV} / \AA)$ & 1.18 & 0.90 & 1.31 & 1.60 & 1.10 & 2.45 \\
\hline \hline
\end{tabular}




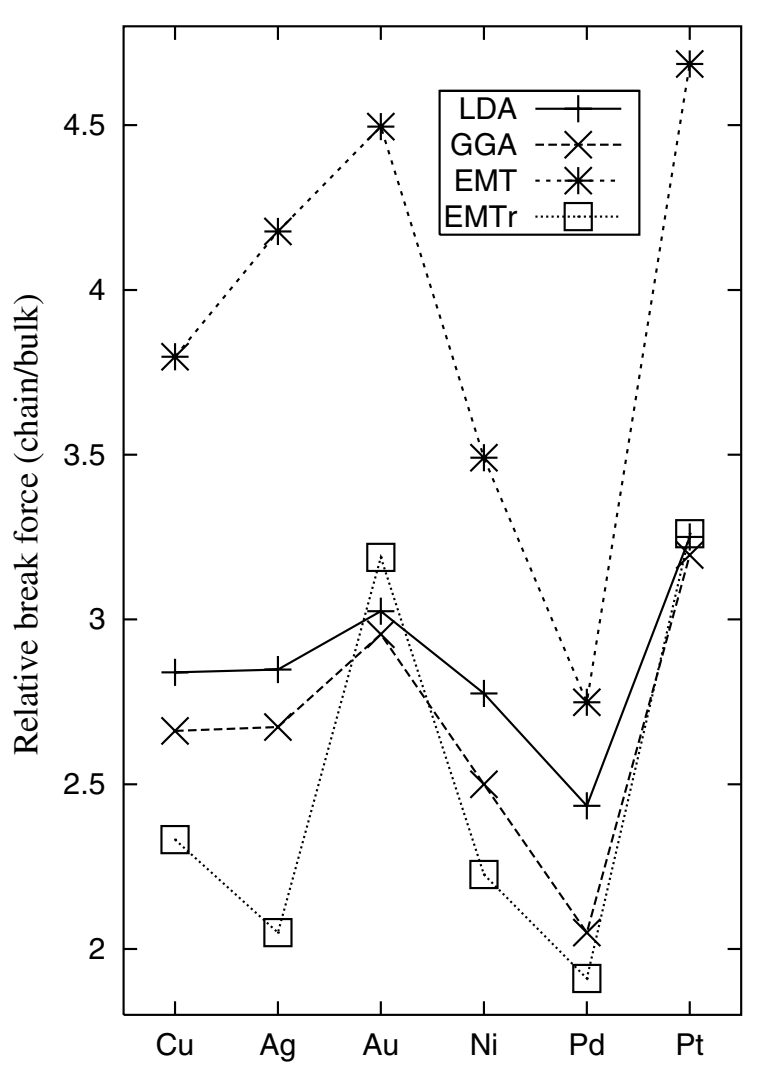

FIG. 3. Calculated break forces for the chains relative to those for bulk crystals for the six metals. The break forces have been calculated within the LDA and GGA approximations and with the EMT potentials. The EMTr calculations include atomic relaxations. Au and Pt clearly stick out with high break forces for the chains.

In conclusion, molecular dynamics simulations indicate that for the six metals $\mathrm{Cu}, \mathrm{Ag}, \mathrm{Au}, \mathrm{Ni}, \mathrm{Pd}$, and Pt the possibility for forming chains in breaking contacts at low temperatures is highest for Au and Pt. This can be understood based on the fact that for these two metals the bonds in low coordinated structures such as chains are very strong relative to bulk bonds as confirmed by DFT calculations.

We would like to thank J.K. Nørskov, J. Schiøtz, G. Rubio-Bollinger, J.M. van Ruitenbeek, and A.I. Yanson for useful discussions on atomic chain formation. The Center for Atomic-Scale Materials Physics (CAMP) is sponsored by the Danish National Research Foundation. The present work was in part financed by the Danish Research Agency through Grant No. 5020-00-0012.

[1] A. I. Yanson, G. Rubio-Bollinger, H.E. van den Brom, N. Agraït, and J. M. van Ruitenbeek, Nature (London) 395, 783 (1998).
[2] H. Ohnishi, Y. Kondo, and K. Takayanagi, Nature (London) 395, 780 (1998).

[3] G. M. Finbow, R. M. Lynden-Bell, and I. R. McDonald, Mol. Phys. 92, 705 (1997).

[4] M. R. Sørensen, M. Brandbyge, and K. W. Jacobsen, Phys. Rev. B 57, 3283 (1998).

[5] A. I. Yanson, University of Leiden, Ph.D. thesis, 2001. After completion of this work we received a preprint reporting new experiments on chain formation [R. H. M. Smit, C. Untiedt, A. I. Yanson, and J. M. van Ruitenbeek, following Letter, Phys. Rev. Lett. 87, 266102 (2001)].

[6] V. Rodrigues, T. Fuhrer, and D. Ugarte, Phys. Rev. Lett. 85, 4124 (2000).

[7] G. Rubio-Bollinger, S. R. Bahn, N. Agraït, K. W. Jacobsen, and S. Vieira, Phys. Rev. Lett. 87, 026101 (2001).

[8] H. Hakkinen, R. N. Barnett, and U. Landman, J. Phys. Chem. B 103, 8814 (1999).

[9] D. Sánchez-Portal et al., Phys. Rev. Lett. 83, 3884 (1999).

[10] L. De Maria and M. Springborg, Chem. Phys. Lett. 323, 293 (2000).

[11] J. A. Torres et al., Surf. Sci. 426, L441 (1999).

[12] M. Okamoto and K. Takayanagi, Phys. Rev. B 60, 7808 (1999).

[13] K. W. Jacobsen, J. K. Nørskov, and M. J. Puska, Phys. Rev. B 35, 7423 (1987); K. W. Jacobsen, P. Stoltze, and J. K. Nørskov, Surf. Sci. 366, 394 (1996).

[14] I. J. Robertson, M. C. Payne, and V. Heine, Europhys. Lett. 15, 301 (1991).

[15] M. Methfessel, D. Hennig, and M. Scheffler, Appl. Phys. A 55, 442 (1992).

[16] K. W. Jacobsen and J. K. Nørskov, in The Structure of Surfaces II, edited by J. F. van der Veen and M. A. van Hove, Springer Series in Surface Sciences Vol. 11 (SpringerVerlag, New York, 1988), p. 118; K. W. Jacobsen, Comments Condens. Matter Phys. 14, 129 (1988).

[17] R. J. Needs, M. J. Godfrey, and M. Mansfield, Surf. Sci. 242, 215 (1991).

[18] Our DFT code uses nonlocal ultrasoft pseudopotentials [19] to describe the ion cores, and the wave functions are expanded in plane waves with kinetic energies up to $400 \mathrm{eV}$ [20]. We have used both the local density approximation (LDA) [21] and the generalized gradient approximation (GGA) [22] for the exchange-correlation energy. The programs used for the calculations are publicly available at http://www.fysik.dtu.dk/campos

[19] D. Vanderbilt, Phys. Rev. B 41, 7892 (1990).

[20] G. Kresse and J. Furthmüller, Comput. Mater. Sci. 6, 15 (1996).

[21] J. P. Perdew and A. Zunger, Phys. Rev. B 23, 5048 (1981).

[22] J. P. Perdew et al., Phys. Rev. B 46, 6671 (1992).

[23] J. H. Rose, J. R. Smith, and J. Ferrante, Phys. Rev. B 28, 1835 (1983).

[24] For the chain a supercell with six atoms has been used while a supercell with $6 \times 6 \times 6$ atoms is used for the calculation of a break force in bulk fcc. The systems are expanded until the points where they become unstable towards small fluctuations. 